Hydraulic Engineering Repository

Ein Service der Bundesanstalt für Wasserbau

Heinzelmann, Christoph; Duric, Zorana; Bödefeld, Jörg Digitalisierung im Verkehrswasserbau

Originalveröffentlichung / Original Publication:

https://doi.org/10.1002/bate.202000029

Verfügbar unter / Available at:

https://hdl.handle.net/20.500.11970/108211

Vorgeschlagene Zitierweise / Suggested citation:

Heinzelmann, Christoph; Duric, Zorana; Bödefeld, Jörg (2020): Digitalisierung im Verkehrswasserbau. In: Bautechnik 97 (6). S. 441-445. https://doi.org/10.1002/bate.202000029. 


\section{Digitalisierung im Verkehrswasserbau}

\begin{abstract}
Die Digitalisierung im Verkehrswasserbau bietet einerseits große Chancen und ist andererseits eine große Herausforderung für die nächsten Jahre. Um das Effizienzpotenzial der Digitalisierung voll auszuschöpfen, muss der gesamte Lebenszyklus der Bauwerke vom Planen und Bauen bis zum Betreiben und Unterhalten betrachtet werden. Unter dieser Voraussetzung ergeben sich Perspektiven, die weit über die Bauwerksplanung und die intelligente Bauwerksmodellierung hinausgehen. Der vorliegende Beitrag präsentiert mögliche Wege für eine erfolgreiche Digitalisierung im Verkehrswasserbau und zeigt auf, welche konkreten Schritte bereits heute unternommen werden können, um eine stabile Basis für eine nachhaltige digitale Entwicklung zu schaffen.
\end{abstract}

Stichworte Wasserstraßen; Wasserbauwerke; Digitalisierung; Lebenszyklus; Prozesse; Daten; Informationsversorgung, nutzerorientiert

\section{Politische Rahmenbedingungen}

In der Vergangenheit sind vermehrt Konflikte bei der Abwicklung von großen Infrastrukturprojekten aufgetreten. Die Folge waren große Abweichungen zwischen den veranschlagten und den abgerechneten Kosten sowie eklatante Überschreitungen des ursprünglich vorgesehenen Terminrahmens. Darunter hat das Vertrauen der Gesellschaft gegenüber den Projektbeteiligten (öffentlicher Bauherr, Genehmigungsbehörde, Ingenieurbüros, Bauindustrie) stark gelitten. Um die Ursachen für diese Fehlentwicklungen $\mathrm{zu}$ analysieren und geeignete Gegenmaßnahmen zu ergreifen, hat das damalige Bundesministerium für Verkehr, Bau und Stadtentwicklung im Jahr 2013 eine Reformkommission eingesetzt, deren Aufgabe es war, die Defizite zu untersuchen und Lösungsvorschläge $\mathrm{zu}$ erarbeiten.

Im Abschlussbericht der Reformkommission des Bundesministeriums für Verkehr und digitale Infrastruktur (BMVI) wurde als Hauptursache für die Fehlentwicklungen ein unzureichendes Kommunikations- und Informationsmanagement genannt [1]. Die Reformkommission empfahl daher, digitale Methoden im gesamten Projektverlauf konsequent $\mathrm{zu}$ nutzen, da sie entscheidend $\mathrm{zu}$ Kostenwahrheit, Kostentransparenz, Effizienz und Termintreue beitragen können [1].

Aufbauend auf den Ergebnissen der Reformkommission hat das BMVI im Jahr 2015 den Stufenplan Digitales Planen und Bauen veröffentlicht [2], für dessen Umsetzung

\begin{abstract}
Digitalisation for hydraulic engineering
On the one hand digitalisation offers great opportunities for hydraulic engineering, while at the same time also being a major challenge for the upcoming years. To fully utilize the efficiency potential of digitalisation, the entire life cycle of structures must be considered: planning, construction, operation and maintenance. Thus opportunities that go far beyond (the digitalisation of) hydraulic structure planning and intelligent hydraulic structure modeling, seem feasible. This article describes possible approaches to successfully digitalizing the field of hydraulic engineering and it goes on to show which steps can be taken to build a foundation for sustainable digital development.
\end{abstract}

Keywords waterways; hydraulic structures; digitalisation; life cycle; processes; data; user-oriented information supply

ein ambitionierter Zeitplan vorgesehen ist. Seitdem arbeiten die Infrastrukturbetreiber daran, die im Stufenplan lediglich grob skizzierten Stufen für ihre Aufgabenbereiche $\mathrm{zu}$ konkretisieren und nach und nach umzusetzen. Als digitale Methode wird im Stufenplan die Methode Building Information Modeling (BIM) vorgegeben. Der Kern von BIM ist ein dreidimensionales Bauwerksmodell mit Bauteilen, die alle relevanten Informationen enthalten $[2,3]$. Ziel ist es, ein virtuelles Abbild der Realität in Form eines digitalen Zwillings zu erschaffen, der sämtliche Informationen über den gesamten Lebenszyklus des Bauwerks aktuell und redundanzfrei vorhält.

Die Wasserstraßen- und Schifffahrtsverwaltung des Bundes (WSV) hat für ihren Aufgabenbereich das Potenzial der Digitalisierung erkannt und für die stufenweise Implementierung der BIM-Methode zwei Pilotprojekte initiiert: den Ersatzneubau der Westkammer der Schleuse Wedtlenstedt und den Neubau der Schleuse Lüneburg. Für das Pilotprojekt Schleuse Wedtlenstedt wurden fünf Anwendungsfälle aus der Planungsphase, z. B. Bestandserfassung und Koordination der Fachgewerke, gewählt. Die Schleuse Lüneburg soll komplett mittels BIM mit einer großen Anzahl von Anwendungsfällen geplant werden. Im Rahmen eines Ziel- und Zukunftskonzepts BIM-WSV 2030 hat die WSV darüber hinaus Projekte ausgewählt, mit denen weitere Anwendungsfälle, insbesondere in der Bau- und Abrechnungsphase, erprobt werden sollen. Allerdings zeigen die bisherigen Projektbearbeitungen, dass die Implementierung der BIM-Methode in der WSV ein langfristiger Prozess sein wird. Dies ist maßgeblich darauf 


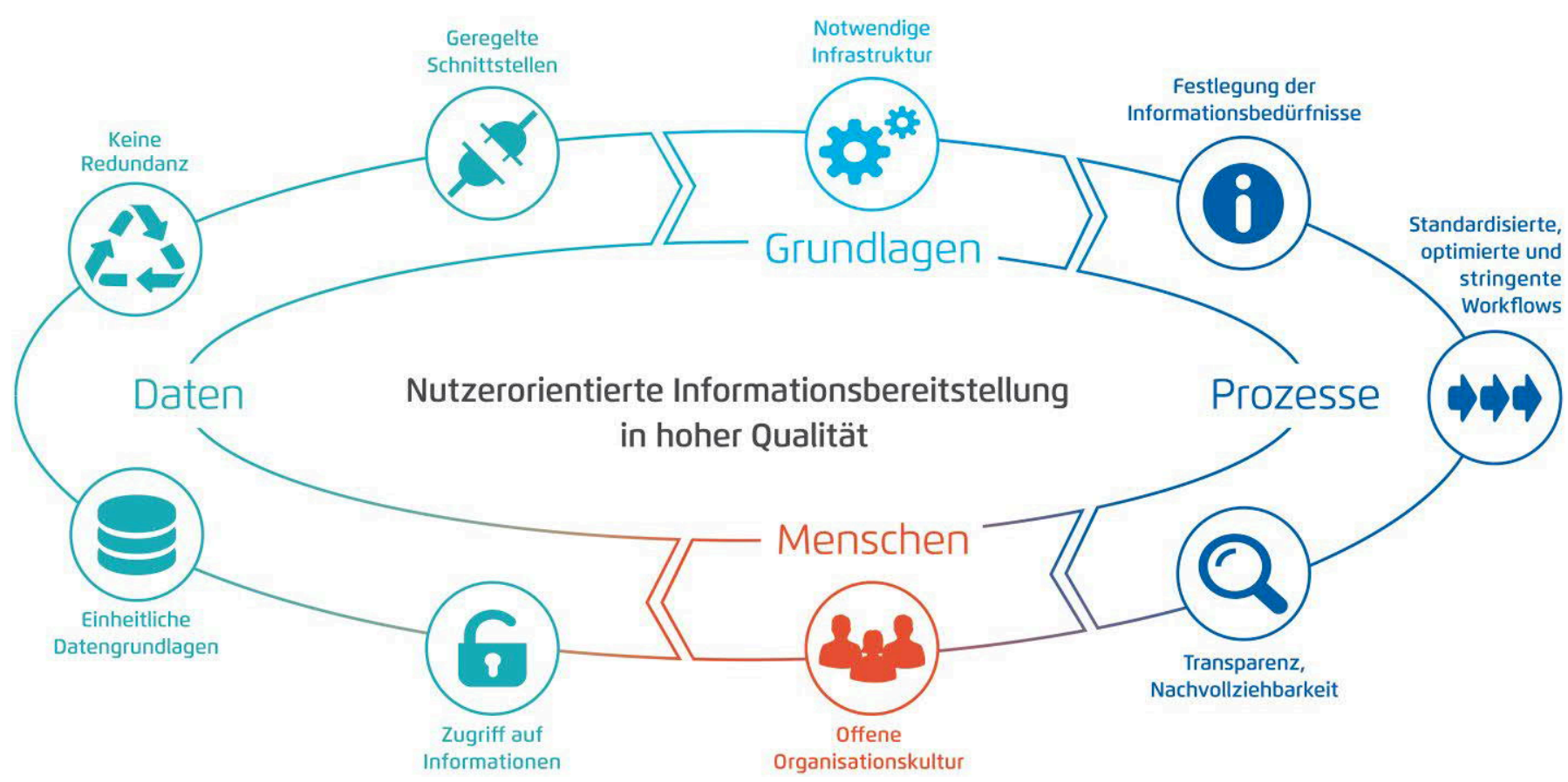

Bild 1 Schematische Darstellung der vier Handlungsfelder (Prozesse, Daten, Menschen und Grundlagen) Schematic representation of the four fields of action (processes, data, people and basics)

zurückzuführen, dass aufgrund der langen Projektlaufzeiten erst nach und nach erste Erfahrungen aus den Pilotanwendungen vorliegen werden.

Auch die Bundesanstalt für Wasserbau (BAW) hat die Digitalisierung im Verkehrswasserbau als wichtige $\mathrm{Zu}-$ kunftsaufgabe aufgegriffen. In ihrer Funktion als technisch-wissenschaftlicher Berater und Gutachter für die WSV entwickelt die BAW einen umfassenden Digitalisierungsansatz für die WSV, der über das digitale Bauwerksmodell hinausgeht. Nachfolgend werden zunächst die Ausgangssituation der Digitalisierung in der WSV und anschließend der Digitalisierungsansatz der BAW sowie die daraus resultierenden Handlungsempfehlungen beschrieben.

\section{2}

\section{Status quo der Digitalisierung in der WSV}

Die WSV setzt bereits heute an vielen Stellen moderne und standardisierte bzw. verbindlich eingeführte IT-Systeme für Planung, Bau und Betrieb von Bauwerken ein. Die IT-Landschaft der WSV ist in den letzten Dekaden stark gewachsen. Im Rahmen einer Bestandserfassung wurden insgesamt 139 aktive IT-Systeme identifiziert, von denen 42 für die Prozesse in den Phasen Planen, Bauen und Betreiben relevant sind [4]. Da die Systeme oft singulär und unabhängig voneinander über Jahre aufgebaut, immer wieder erweitert und dem jeweiligen Bedarf angepasst wurden, ist ein Mosaik zahlreicher Anwendungen und Datenbanken mit technologischer Heterogenität und redundanten Daten in unterschiedlicher Qualität entstanden.
Die Herausforderung im Zuge der weiteren Digitalisierung besteht darin, die vorhandenen IT-Systeme zu vernetzen, um redundante Daten zu vermeiden, die Datenqualität zu verbessern und die Daten auch außerhalb der originären IT-Systeme nutzbar zu machen.

\section{$3 \quad$ Handlungsfelder der Digitalisierung}

Der von der BAW verfolgte Digitalisierungsansatz setzt sich aus den folgenden vier Handlungsfeldern zusammen: Prozesse, Daten, Menschen und Grundlagen (Bild 1). Nachfolgend werden die einzelnen Handlungsfelder beschrieben und die zugehörigen Ziele erläutert.

\subsection{Prozesse}

Um die Informationsbedürfnisse der in einem Bauprojekt Beteiligten identifizieren zu können, müssen die Prozesse bekannt und eindeutig beschrieben sein. Ein Prozess ist „ein Satz zusammenhängender (...) Tätigkeiten, der Eingaben zum Erzielen eines vorgesehenen Ergebnisses verwendet" [5]. Einzelne Prozesse, die in den verschiedenen Lebensphasen eines Bauwerks vorkommen, z. B. die Erstellung einer Voruntersuchung zu Projektbeginn, sind bereits durch Verwaltungsvorschriften und andere Regelwerke dokumentiert. Jedoch ist häufig eine weitere Konkretisierung erforderlich, was z.B. den Umfang, die Art der Unterlagen und explizite Entscheidungsmomente betrifft. Eine systematische Untersuchung der entsprechenden Prozesse ermöglicht, neben der Abfolge der Aktivitäten und den beteiligten Rollen, die Entscheidungsmomente und die dafür benötigten Informationen zu ermitteln. 
Vorrangiges Ziel im Handlungsfeld Prozesse ist es, die Prozesse und IT-Systeme zu standardisieren. Die Standardisierung der Prozesse sowie deren Implementierung in die bestehende IT-Landschaft bieten die Chance, eine höhere Prozesstransparenz und -struktur zu schaffen, mit der auch eine Optimierung der Prozessleistung einhergeht. Des Weiteren kann der Koordinationsaufwand gesenkt und die Leistungseffizienz gesteigert werden. Die Voraussetzung ist eine verbindliche Nutzung der standardisierten Prozesse und IT-Systeme.

\subsection{Daten}

Voraussetzung für die bedarfsorientierte Bereitstellung der Informationen ist ein einheitliches Informationsmodell. Dieses Modell beinhaltet strukturierte und qualitativ hochwertige Daten für einen effektiven Zugriff und eine einheitliche Datengrundlage. Da bereits heute zahlreiche Daten in verschiedenen IT-Systemen digital vorliegen, ist es aus Akzeptanz- und Ressourcengründen sinnvoll, diese Systeme zu nutzen und in das Informationsmodell $\mathrm{zu}$ integrieren. Dies erfordert, dass die Daten aus den verschiedenen IT-Systemen miteinander vernetzt werden. Voraussetzung dafür ist neben der Datenharmonisierung und -bereinigung auch die Regelung der Schnittstellen zum Datenimport und -export zwischen den IT-Systemen.

Ziel im Handlungsfeld Daten ist es, qualitätsgesicherte und strukturierte Daten zu schaffen. Die Qualität bezieht sich dabei u. a. auf die Richtigkeit und Vollständigkeit, die Verständlichkeit sowie die Aktualität der Daten. Eine einheitliche strukturierte Ablage gewährleistet in Kombination mit einer zeitgemäßen IT eine bessere Zugänglichkeit bzw. schnelle Auffindbarkeit, was ein weiteres Qualitätsmerkmal von Daten ist. Werden die Daten im Rahmen von Prozessen genutzt, erfolgt die Pflege automatisch. Eine manuelle Nachpflege entfällt.

\section{3}

\section{Menschen}

Bei allen Schritten, die im Zuge der Digitalisierung gegangen werden, ist die Beteiligung und Mitnahme der betroffenen Menschen von entscheidender Bedeutung. Dies reicht von der Information der Beteiligten über Möglichkeiten des Erfahrungsaustauschs bis hin zur Förderung neuer Ideen und Initiativen.

Ziel im Handlungsfeld Menschen ist es, die betroffenen Menschen frühzeitig in den Veränderungsprozess einzubinden, sie durch eine offene Organisationskultur für die Anwendung digitaler Werkzeuge zu motivieren und $\mathrm{zu}$ qualifizieren.

\section{Grundlagen}

Ziel im Handlungsfeld Grundlagen ist die Bereitstellung einer leistungsfähigen und zeitgemäßen IT-Infrastruktur.
Dazu zählen eine zuverlässige Hard- und Software sowie eine stabile und leistungsfähige Netzwerkanbindung.

\section{$4 \quad$ Handlungsempfehlungen für die Digitalisierung}

Für die Erreichung der für die einzelnen Handlungsfelder formulierten Ziele werden Handlungsempfehlungen gegeben (Tab. 1). Die möglichen Maßnahmen reichen von konkreten, schnell umzusetzenden Schritten bis hin zu Entwicklungen, die jetzt anzustoßen sind und eine längere Perspektive aufweisen.

Die Digitalisierung betrifft alle Bereiche einer Organisationseinheit und einer Wertschöpfungskette. Die Handlungsfelder Prozesse, Daten, Menschen und Grundlagen müssen gleichermaßen in den digitalen Transformationsprozess einbezogen werden. Besonders in großen Organisationen ist diese digitale Veränderung komplex und verlangt ein visionäres und strategisches Handeln. Der digitale Wandel für den Verkehrswasserbau sollte daher fachübergreifend gesteuert werden. Daher wird empfohlen, eine eng an die Organisationsführung angebundene Digitalisierungskoordination zu gründen.

\subsection{Prozesse}

Das Handlungsfeld Prozesse erfordert eine detaillierte Analyse der Prozesse, was zunächst für kleinere Bauprojekte erprobt werden sollte. Mögliche Beispiele für die $\mathrm{zu}$ analysierenden Prozesse sind: die Erstellung einer Voruntersuchung, die Aufstellung von Entwurfsunterlagen für die Haushaltsanmeldung sowie die Übergabe der Bestandsunterlagen an die für den Betrieb und die Unterhaltung verantwortlichen Stellen. Im Rahmen von Workshops oder Befragungen mit Projektbeteiligten lassen sich die Abhängigkeiten und die Aktivitäten des betrachteten Prozesses identifizieren. Darüber hinaus sind relevante Informationen aus Projektunterlagen und Regelwerken zu berücksichtigen. Eine Visualisierung der Ergebnisse in Form von Ablaufdiagrammen, wie beispielhaft in Bild 2 dargestellt, sichert die eindeutige Beschreibung der Prozesse. Ein Vergleich der Ergebnisse aus verschiedenen Projekten und eine Vereinheitlichung sowie perspektivisch eine Standardisierung der Prozesse können in einem Folgeschritt stattfinden. Durch ein kontinuierliches Anwender-Feedback wird die Möglichkeit gegeben, die Prozesse schrittweise zu verbessern

\subsection{Daten}

Für das Handlungsfeld Daten sind die im Rahmen der Bestandserfassung der IT-Systeme identifizierten Optimierungspotenziale umzusetzen. Dazu zählen z.B. die Realisierung einer verbindlichen Nutzung von eingeführten IT-Systemen, die Implementierung einer einheitlichen Bauteilstruktur in den IT-Verfahren und die Optimierung 
Tab. 1 Handlungsempfehlungen

Recommendations for action

\begin{tabular}{ll}
\hline Handlungsfeld & Handlungsempfehlungen \\
\hline Prozesse & Detaillierte Analyse einzelner Prozesse \\
Daten & Umsetzung der Optimierungspotenziale aus der Bestandserfassung der IT-Landschaft: \\
& - Einführung einer verbindlichen Nutzung der IT-Systeme \\
& - Vereinheitlichung von Bauteilstrukturen \\
& - Optimierung des digitalen Baubestandwerks \\
& - Aufbau einer Preisdatenbank \\
& - Modernisierung und Optimierung des digitalen Objektkatasters \\
& - Aufbau eines zentralen Projektinformationssystems
\end{tabular}

Menschen Bereitstellung digitaler Werkzeuge für Kommunikation, Einrichtung eines Innovation-Labs

Grundlagen Bereitstellung von technischen Standardwerkzeugen für alle Dienststellen (z. B. flächendeckender Netzwerkzugang, digitale Kommunikationswerkzeuge)

Alle Felder Etablierung einer eng an die Organisationsführung angebundenen Digitalisierungskoordination

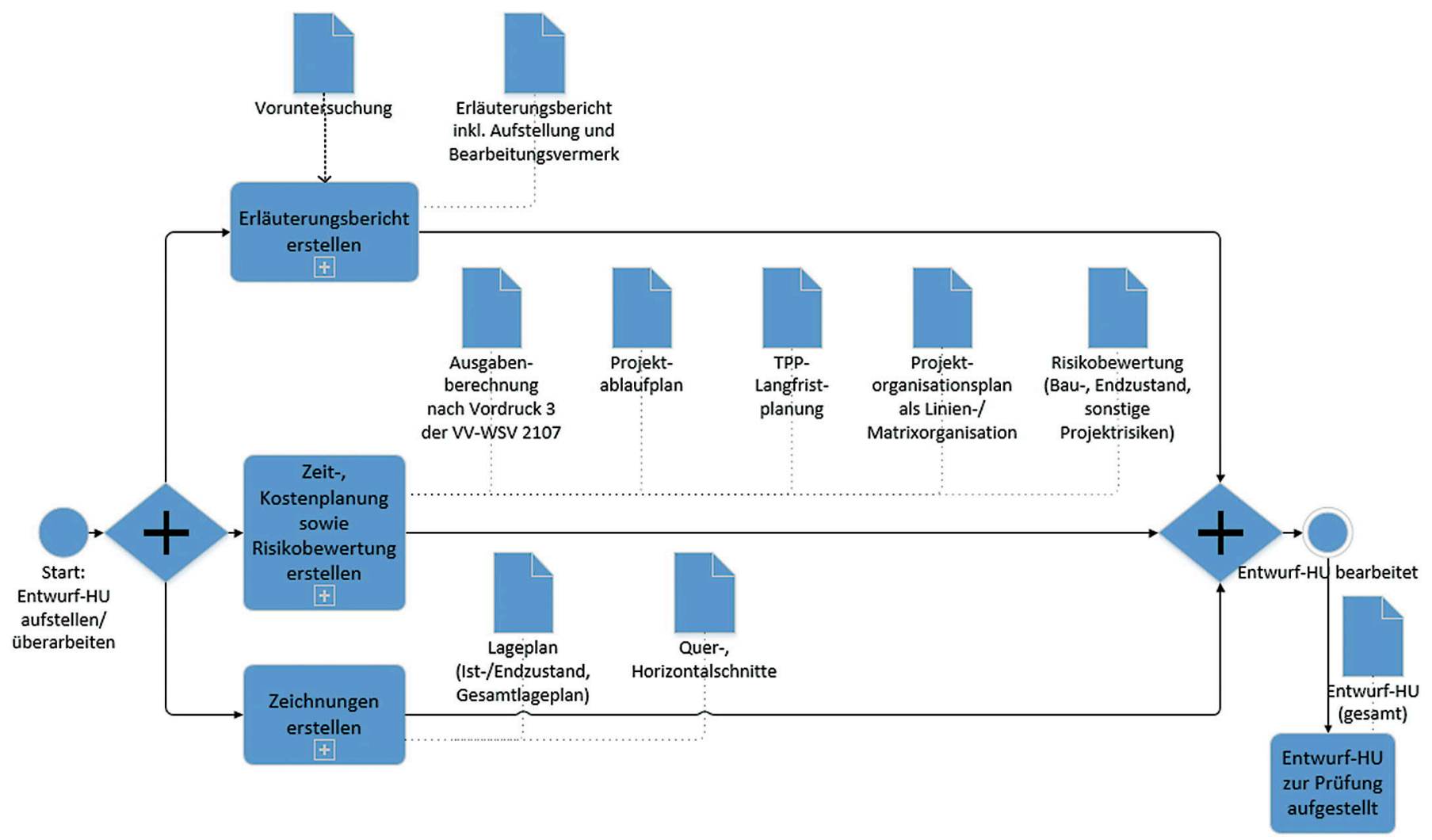

Bild 2 Auszug aus dem Prozessdiagramm für den Entwurf Haushaltsunterlage (Rolle: Projektleitung), beispielhaft Extract from the process diagram for the design of the budgetary framework (role: project management), exemplary

des digitalen Baubestandwerks im Hinblick auf einen verbesserten Informationszugang. Ferner ist eine Preisdatenbank aufzubauen.

Die Bestandserfassung zeigt, dass das Kernsystem für das Management der Wasserstraßeninfrastruktur mit ihren über 50000 Bauwerken das digitale Objektkataster ist. Aktuell realisiert die WSV ein neues Objektkataster [4]. Neben der Bereitstellung qualitativ hochwertiger Daten in leicht recherchierbarer, nutzerorientierter Form ermöglicht eine eindeutige Objektidentifizierung die Kopplung mit anderen IT-Systemen. Somit bildet das neue Ob- jektkataster als Rückgrat für alle Informationssysteme das digitale Fundament für ein nachhaltiges Infrastrukturmanagement.

Eine aktuelle und umfassende Übersicht über den Stand von laufenden Projekten ist ein wichtiger Bestandteil eines erfolgreichen Infrastrukturmanagements. Aus diesem Grund entsteht derzeit ein zentrales Projektinformationssystem, das einen Überblick und eine Auswertungsmöglichkeit aller aktuellen, priorisierten Bauprojekte mit relevanten Informationen für die Steuerungsebene der WSV bietet [4]. 


\section{3}

Menschen

In der WSV werden bereits einzelne Digitalisierungsansätze und neue Technologien erprobt. Um das gewonnene Wissen für einen größeren Personenkreis in der WSV nutzbar zu machen, ist ein stärkerer Austausch erforderlich. Neben Aussprachetagen und Workshops wird die koordinierte Nutzung neuer Austauschformate, wie z.B. Lernvideos, Online-Foren oder Blogs, empfohlen. Sie ermöglichen einen zeit- und ortsunabhängigen Zugriff auf das spezielle Wissen. Eine weitere Empfehlung im Handlungsfeld Menschen ist die Einrichtung eines InnovationLabs, das die Beschäftigten bei der Erprobung neuer moderner Werkzeuge unterstützt. Damit können Erfahrungen schnell und einfach gesammelt und die Technologie hinsichtlich weiterer Nutzung bewertet werden.

\subsection{Grundlagen}

Das Handlungsfeld Grundlagen fordert einen flächendeckenden, bedarfsgerechten Netzwerkzugang, auch entlang der Wasserstraßen. Darüber hinaus ist für die effektive, ortsunabhängige Zusammenarbeit die Bereitstellung digitaler Kommunikationswerkzeuge erforderlich, z. B. in Form von Webkonferenzen.

\section{Zusammenfassung}

Die Digitalisierung schreitet in allen Lebensbereichen mit großer Dynamik voran und bietet für Planen, Bauen und

\section{Literatur}

[1] BMVI (2015) Reformkommission Bau von Großprojekten. Komplexität beherrschen - kostengerecht, termintreu und effizient. Berlin: Bundesministerium für Verkehr und digitale Infrastruktur.

[2] BMVI (2015) Stufenplan Digitales Planen und Bauen. Einführung moderner, IT-gestützter Prozesse und Technologien bei Planung, Bau und Betrieb von Bauwerken. Berlin: Bundesministerium für Verkehr und digitale Infrastruktur.

[3] Borrmann, A.; König, M.; Koch, Ch.; Beetz, J. (2015) Building Information Modeling, Technologische Grundlagen und industrielle Praxis. Wiesbaden: Springer Vieweg.
Betreiben der Wasserstraßen große Chancen. Damit der Verkehrswasserbau von dem digitalen Wandel profitieren kann, bedarf es einer klar definierten Digitalisierungsstrategie.

Für einen umfassenden Digitalisierungsansatz hat die BAW vier Handlungsfelder identifiziert: Prozesse, Daten, Menschen und Grundlagen. Für jedes dieser Handlungsfelder wurden Ziele und Handlungsempfehlungen erarbeitet. Neben konkreten Empfehlungen, wie z.B. die Einrichtung einer Digitalisierungskoordination zur fachübergreifenden Steuerung, die Etablierung eines neuen digitalen Objektkatasters als Rückgrat der Informationsmodelle sowie die Einrichtung eines zentralen Projektinformationssystems, wurden auch perspektivische Empfehlungen formuliert, wie z.B. die stärkere Standardisierung der Prozesse für Planen, Bauen und Betreiben der Wasserstraßeninfrastruktur. Die formulierten Ziele und Empfehlungen bilden eine wichtige Grundlage für die erfolgreiche Digitalisierung im Verkehrswasserbau.

\section{Anmerkung der Autoren}

Das vorliegende Manuskript ist bereits unter dem Titel „Wege zur Digitalisierung im Verkehrswasserbau“, Tagungsband des 43. Dresdner Wasserbaukolloquiums, 5./6. März 2020, S. 11-20 erschienen und wurde lediglich geringfügig aktualisiert.

[4] Schenk, D.; Laursen, Ch. (2020) Digitalisierung im Verkehrswasserbau: Aktuelle Entwicklungen in: Tagungsband des 43. Dresdner Wasserbaukolloquiums. Dresden, 5./6. März 2020. S. 89-98.

[5] DIN EN ISO 9000:2015-11 (2015) Qualitätsmanagementsysteme - Grundlagen und Begriffe (ISO 9000:2015). Berlin: Beuth. Ausgabe Nov. 2015.

Dr.-Ing. Zorana Duric (Korrespondenzautorin)

zorana.duric@baw.de

Bundesanstalt für Wasserbau

Kußmaulstraße 17

76187 Karlsruhe

\section{Zitieren Sie diesen Beitrag}

Heinzelmann, C.; Bödefeld, J.; Duric, Z. (2020) Digitalisierung im Verkehrswasserbau. Bautechnik 97, H. 6, S. 441-445.

https://doi.org/10.1002/bate.202000029 